\title{
Gli autori
}

Ugo Bardi è professore associato di Chimica fisica, Università di Firenze.

Emidio D'Angelo è economista dell'energia presso l'Osservatorio Energia, IRES Cgil.

Roberto Giulianelli è ricercatore in Storia economica, Università Politecnica delle Marche.

Marco Giovagnoli è ricercatore in Sociologia dei Processi economici e del Lavoro, Università di Camerino.

Andrea Masullo è professore a contratto di Fondamenti di Sostenibilità ambientale, Università di Camerino.

Gianni Mattioli è professore associato di Fisica, Università di Roma "La Sapienza". Antonio Minetti è dirigente Servizio ambiente e paesaggio, Regione Marche.

Fabio Polonara è professore ordinario di Fisica tecnica e industriale, Università Politecnica delle Marche.

Serena Rugiero è coordinatrice dell'Osservatorio Energia, IRES Cgil.

Andrea Prontera è professore a contratto di Relazioni internazionali e Studi strategici, Università di Macerata.

Massimo Scalia è professore associato di Fisica matematica, Università di Roma "La Sapienza".

Gianni Silvestrini è ingegnere e direttore scientifico del Kyoto Club.

Hanno commentato questo numero:

Riccardo Bilancioni, imprenditore marchigiano.

Maurizio Di Cosmo, segretario regionale CGIL Marche.

Claudio Falasca, coordinatore del Dipartimento Ambiente e Territorio, CGIL. Assemblea Permanente NoCentraliAPI. 